DOI:

\title{
Development and Characterization of Conjugated Polyaniline/Co Doped ZnO Nanocomposites for Enhanced Dye Oxidation from Wastewater
}

\author{
Belal A. Tahoun ${ }^{1,2}$, Elsayed M. Farag ${ }^{2}$, Shehab A. Mansour ${ }^{1,2}$, Maha A. Tony ${ }^{1,2}$ \\ ${ }^{1}$ Advanced Materials/Solar Energy and Environmental Sustainability (AMSEES) Laboratory, Faculty of \\ Engineering, Menoufia University, 32511 Shebin El-Kom, Egypt \\ ${ }^{2}$ Basic Engineering Science Department, Faculty of Engineering, Menoufia University, 32511 Shebin El- \\ Kom, Egypt \\ (Corresponding author: ele.engbelal@gmail.com)
}

\begin{abstract}
Polymer nanocomposites based on wide band gap metal oxide nanoparticles are promised functional nanomaterials in many potential applications due to their facile processability. One of their most potential applications their capability of utilization in the field of photocatalytic oxidation technology. In this respect, fabrication of polyaniline (PANI) nanocomposites based on Co-doped $\mathrm{ZnO}$ nanorods is introduced. The Codoped $\mathrm{ZnO}$ nanorods were synthesized using hydrothermal route with molar ratio between cobalt and zinc precursor salts reach to 0.07. XRD and UV-visible absorption measurements confirmed the formation of wurtzite $\mathrm{ZnO}$ nanostructured with substitution of $\mathrm{Zn}$ sites by $\mathrm{Co}$ ions in $\mathrm{ZnO}$ host structure. The morphological investigation of PANI/Co-doped $\mathrm{ZnO}$ nanocomposites using HR-TEM confirmed the interaction between the synthesized nanorods and PANI matrix. In the present study, the oxidation of synthetic textile dying effluent containing methylene blue has been carried out via PANI/Co-doped ZnO nanocomposites. The attained nanocomposite samples in various Co concentrations labelled as P-CZ0, PCZ1, P-CZ3, P-CZ5 and P-CZ7 were checked for their catalytic activity for wastewater treatment under the UV-illumination. The influence of various operating parameters, i.e., circulation flow rate, initial catalyst concentration, initial dye loading and $\mathrm{pH}$ for the three systems, was investigated, and the optimal operating conditions were recorded. The experimental results revealed that increasing the dye load is reducing the catalytic activity of the dye mineralization. Also, the circulation flow rate is optimized at $147 \mathrm{~mL} / \mathrm{min}$ at $\mathrm{pH}$ 6.0 for all systems. The experimental data confirms the PANI/ Co-doped ZnO possess the highest catalytic activity which could oxidize $84 \%$ of wastewater with a minimum catalyst use of $20 \mathrm{mg} / \mathrm{L}$. The success of this method explores the opportunity of combined photocatalyst in increasing the catalytic activity for higher dyecontained wastewater oxidation. Thus, such advantage could expand the application of the system as an oxidation technique for wastewater treatment.
\end{abstract}

\section{Keywords: Nanocomposites, Co-doped ZnO, Conducting Polymers, Photocatalysis}

\section{Introduction}

Recently, hazardous pollutants of emerging concern could be identified in air and water affect human beings, animals, vegetation and the environment. Organic pollutants, especially dyes, for industrial wastewater cause many problems to human and soil. The result is the wastewater drained to rivers or any other watercourse that may damage or affect the agriculture. Also, such wastewater could be drained to the groundwater that causes a severe damage to the soil to be toxic. Hence, there is an urgent need for worldwide coordinated research aiming to pollutant detection, assessment, and abatement, especially concerning new extremely harmful emerging contaminants [1].
Various conventional physical/chemical or may be biological treatment technologies are introduced to eliminate dyes from aqueous effluents. However, those conventional technologies such as filtration, membrane separation and adsorption are unfavorable due to their high treatment cost or they may be transferring the pollutants from one phase to another without mineralizing them [2].

Photocatalytic oxidation technology, is signified as one of Advanced Oxidation Technologies (AOTs), that has been emerged as superior efficient reactions [3]. Photocatalytic oxidation is based on the formation of high reactive groups, namely ${ }^{\circ} \mathrm{OH}$ radicals, which are responsible of oxidizing and mineralizing such pollutants to harmless end 


\section{Belal A. Tahoun, Elsayed M. Farag, Shehab A. Mansour and Maha A. Tony" Development and Characterization of Conjugated Polyaniline/Co doped ZnO Nanocomposites}

products, i.e. $\mathrm{CO}_{2}, \mathrm{H}_{2} \mathrm{O}$, and inorganic matter [4, 5]. Numerous catalysts have been introduced as a source of catalytic oxidants, e.g. $\mathrm{ZnO}[6], \mathrm{TiO}_{2}[7,8]$ and iron oxides $[9,10]$. Such materials are subjected as photocatalyst for wastewater oxidation and completely mineralizing organic pollutants. However, searching for novel coupled materials with sufficient treatment capabilities and innovative characteristics is attaining the scientists' attention. The photocatalytic activity of any photocatalyst is dependent on surface and structural properties which include crystal phase structure, surface area, particle size distribution, porosity and band gap energy [11]. Out of all semiconductors, $\mathrm{ZnO}$ arises as a superior candidate among the photocatalyst for its use in green remediation due to its unique properties. $\mathrm{ZnO}$ possess a wide band gap in the near-UV spectral region, good photocatalytic property, strong oxidation capability, insoluble in water, and low-cost production $[12,13]$.

Searching to improve the catalytic activity of such catalysts are the interest of the scientist. The addition of impurities to a very pure substance is known as doping, which is divided into the following two categories: (1) cationic (metal) doping and (2) anionic (nonmetal) doping. In cationic doping, the semiconductor is doped with cations, for example $\mathrm{Al}, \mathrm{Cu}, \mathrm{V}, \mathrm{Cr}, \mathrm{Fe}, \mathrm{Ni}, \mathrm{Co}, \mathrm{Mn}$, and so on, while in anionic doping, anions are used, for example N, S, F, $\mathrm{C}$, and so on. Among the transition metal ions, Co and $\mathrm{Zn}$ doping elements show considerable interest in the scientific community due to their unique characteristics.

In the last few decades, the conducting polymers have much interest in various potential fields such as charge storage materials, catalysts, and optoelectronic devices. Conducting polymers are consisted of $\pi$ conjugated system and offer unusual electronic properties such as electrical conductivity, low energy optical transitions, low ionization potential, and high electron affinity. Polyaniline (PANI) is one of the most important and popular conducting polymers due to its unique electrical and photoelectric characteristics which could be easily altered either by the oxidation of PANI chain or by the protonation of imine nitrogen backbone [14]. PANI shows high environmental stability and displays process feasibility.

The preparation of nanocomposites of PANI with inorganic nanoparticles is thought to be a potential route to improve the performance of PANI aiming to obtain the materials with synergetic or complementary behavior between PANI and inorganic nanoparticles [15]. PANI nanocomposites based on $\mathrm{ZnO}$ and $\mathrm{AL}$-doped $\mathrm{ZnO}$ have been emerged quite good results through their usage in AOT under visible light as reported in degradation of methylene orange and rose bengal, respectively [16]. Moreover, $\mathrm{PANI} / \mathrm{ZnO}$ nanocomposites have been exhibited a good photocatalytic effect on MB $(75 \%$ degradation after 4 hours for UV light and $95 \%$ degradation after $4 \mathrm{~h}$ under sun light) and MG dyes degradation under UV and natural sunlight irradiation [17]. Doping of $\mathrm{ZnO}$ with $\mathrm{Co}$ [18] and coupling with PANI extends the absorbing region to the visible spectrum [19]. This makes the Co-doped ZnO/PANI material novel catalyst. In the present study PANI/ Co-doped $\mathrm{ZnO}$ samples with different molarities between $\mathrm{Co}$ and $\mathrm{Zn}$ precursor salts were prepared and the system was studied under the UV-illumination to oxidize wastewater contaminated with methylene blue (MB) dye.

\section{Material and methods 2.1 Fabrication of the investigated polyaniline nanocomposites}

The investigated PANI nanocomposites in the present work are fabricated using direct oxidation polymerization process. The reagents of the polymerization process were of the analytical grade without additional purification. Aniline (Aldrich, Germany), ammonium persulfate (APS from bioWORLD, India) and hydrochloric acid (HCL $37 \%$, spectrum, India) were used in PANI polymerization process. The polymerization process was done in an acidic solution using APS as a chemical oxidant in presence of Co-doped $\mathrm{ZnO}$ nanorods. For more elucidations on the used fabrication method, Fig. (1) was depicted the details of fabrication.

Figure (1a) shows the schematic diagrams for the used hydrothermal synthetization processes of the Co-doped $\mathrm{ZnO}$ nanorods. Whereas Fig. (1b) is shown the schematic diagram for the PANI nanocomposites polymerization process in presence of the desired amount of Co-doped $\mathrm{ZnO}$ nanorods, weight ratio of Co-doped $\mathrm{ZnO}$ to aniline was 7:93\%, using chemical oxidation method. The weight percentage ratio between the used $\mathrm{Co}$ and $\mathrm{Zn}$ precursor salts as well as the corresponding name of each nanocomposite are listed in Table (1). However, the obtained nanocomposite samples were labelled as P-CZ0, P-CZ1, P-CZ3, P$\mathrm{CZ5}$ and $\mathrm{P}-\mathrm{CZ7}$ according to the utilized molarity concentration of $\mathrm{Co}\left(\mathrm{NO}_{3}\right)_{2} \cdot 6 \mathrm{H}_{2} \mathrm{O} ; 0,1,3,5$ and $7 \%$, respectively.

\subsection{Photooxidation experiments}

In order to evaluate the photocatalytic activity of allsynthesized samples on MB dye, a simple photochemical reactor is used. The system is consisting of ultraviolet source, dosing pump (Model: $\mathrm{BL} 5,5 \mathrm{~L} / \mathrm{H}$ and 7 bar) and $250 \mathrm{~mL}$ beaker containing the dye and photocatalyst, which are well mixed 


\section{Belal A. Tahoun, Elsayed M. Farag, Shehab A. Mansour and Maha A. Tony" Development and Characterization of Conjugated Polyaniline/Co doped ZnO Nanocomposites

using magnetic stirrer. The schematic diagram of experiment is shown in Fig. (2). The present of magnetic stirrer to ensure mixing and dispersion of dye and photocatalyst during experiment period. Moreover, pH-meter (Adwa AD1030 pH/mV \& Temperature meter), is used to adjust the $\mathrm{pH}$ of the solution, if required, prior to the solution is subjected to the UV-illumination.

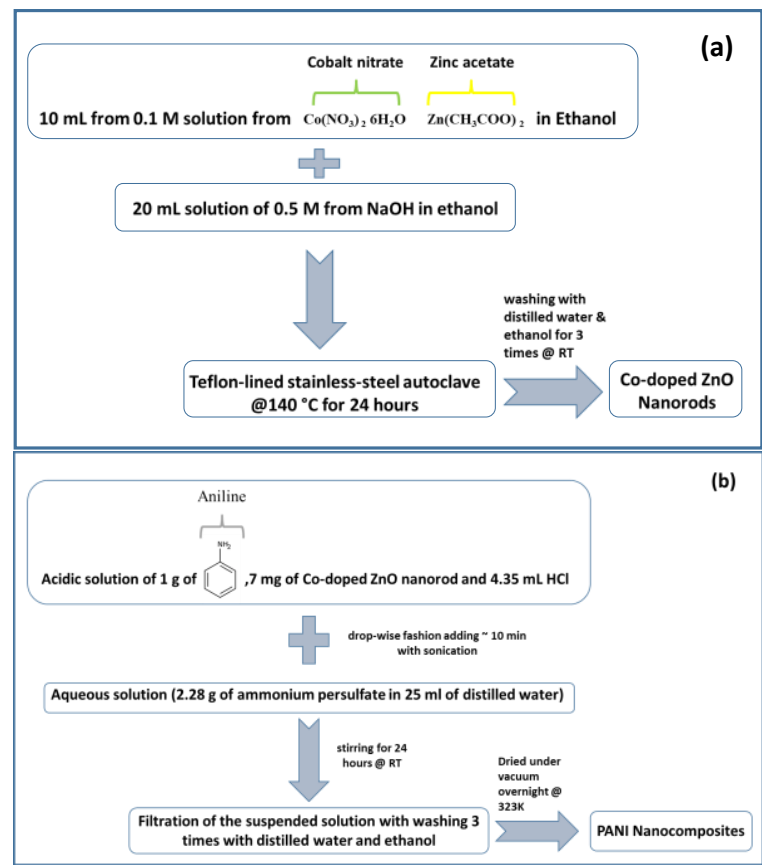

Fig. (1) Schematic diagram preparation of PANI/Codoped $\mathrm{ZnO}$ nanocomposites, (a) Schematic diagram for hydrothermal process of synthetization of Codoped $\mathrm{ZnO}$ nanorods, (b) Schematic diagram for PANI Nanocomposites polymerization process in the presence of $\mathrm{Co}$-doped $\mathrm{ZnO}$ nanorods.

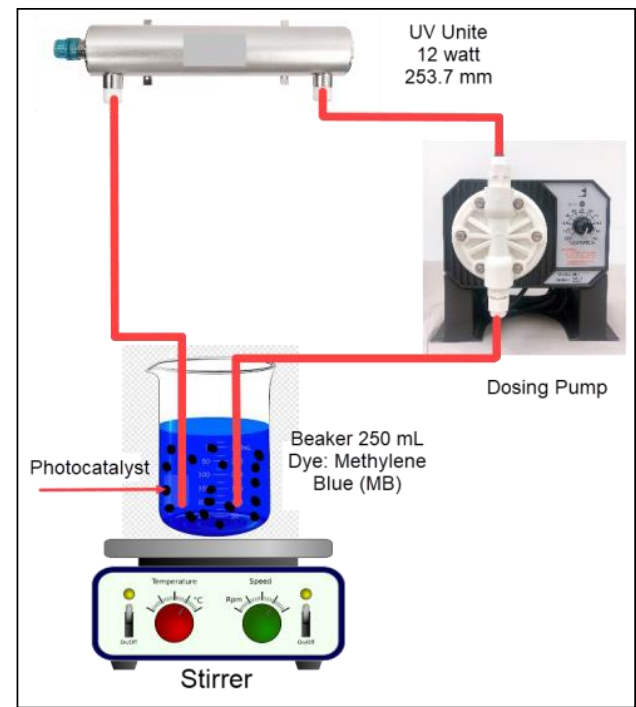

Fig. (2): Schematic representation of the photochemical experiment setup
3. Results and discussion

3.1 Characterization of Co-doped $\mathrm{ZnO}$ nanorods and polyaniline nanocomposites

The microstructure characterization of the investigated Co-doped $\mathrm{ZnO}$ nanorods using $\mathrm{X}$-ray diffraction (XRD) confirmed the formation of pure wurtzite hexagonal phase $\mathrm{ZnO}$ phase without any phases that may be related to other phases of $\mathrm{ZnO}$ and/or cobalt. Such results confirm the stability of the host structure of $\mathrm{ZnO}$ crystal although of the addition of Co ion due to the substitution of the $\mathrm{Zn}$ sites by Co that is usually suggested for low concentration of transition metal ions [20].

Table (1): Cobalt acetate molarity \%, calculated particle size (D) using highest three diffraction XRD pattern, calculated optical energy gab (Eg)

\begin{tabular}{cccccc}
$\begin{array}{c}\text { Sample } \\
\text { Name }\end{array}$ & CZ0 & CZ1 & CZ3 & CZ5 & CZ7 \\
\hline $\begin{array}{c}\text { Cobalt } \\
\text { acetate } \\
\text { molarity\% }\end{array}$ & 0 & 1 & 3 & 5 & 7 \\
$\mathrm{D}(\mathrm{nm})$ & 40.43 & 38.31 & 40.34 & 35.29 & 44.85 \\
$\mathrm{Eg}(\mathrm{eV})$ & 3.034 & 3.016 & 3.112 & 3.138 & 3.152 \\
& & & & & \\
$\boldsymbol{c} / \boldsymbol{a}$ & 1.603 & 1.604 & 1.603 & 1.602 & 1.605
\end{tabular}

The crystallite size of the investigated Co-doped $\mathrm{ZnO}$ samples was calculated from the full width at half maximum (FWHM) of the XRD peaks using Scherer formula [21-23], $D=(0.9 \lambda) /\left(\beta_{\mathrm{hkl}} \cos \theta\right)$, where $\beta_{\mathrm{hkl}}$ is FWHM of the XRD peak and $\theta$ the diffraction angle at (hkl) plane, and $\lambda$ is the wavelength of the $\mathrm{X}$ ray source. The crystallite size (D) was calculated by using such formula for the highest three diffracted peaks which corresponding to the planes: (100), (002), and (101) and the obtained values are listed in Table (1). The values of the lattice constants (c \& a) and c/a ratio were estimated for the investigated Codoped $\mathrm{ZnO}$ samples using this relation; $\frac{1}{d_{h k l}^{3}}=$ $\frac{4}{3}\left(\frac{h^{2}+h k+k^{2}}{a^{2}}\right)+\frac{l^{2}}{c^{2}}$ of hexagonal structure. The obtained c/a ratio of the investigated Co-doped $\mathrm{ZnO}$ samples were very close as illustrated in Table (1). The obtained c/a ratio for the studied samples was varied from 1.602 to 1.605 with average 1.6034 . These results are in well agreement with those given in the standard data $(\mathrm{c} / \mathrm{a}=1.6023)$ according to card (JCPDS-36-1451) [24]. The obtained values of lattice constants for all studied Co-doped $\mathrm{ZnO}$ samples are very close that indicates the substitution of $\mathrm{Zn}$ ions 


\section{Belal A. Tahoun, Elsayed M. Farag, Shehab A. Mansour and Maha A. Tony" Development and Characterization of Conjugated Polyaniline/Co doped ZnO Nanocomposites}

by Co ions are replacing without pronounced change in the volume of the unite cell $[20,25]$.

In order to measure the UV-Vis absorbance of Codoped $\mathrm{ZnO}$, the synthesized nanorods were dispersed in ethanol with $1 \mathrm{mg} / \mathrm{ml}$ concentration for each sample. The absorbance peak for all measured samples varies from 374 to $362 \mathrm{~nm}$ with blue shift with increasing the Co concentration as shown in Fig. (3a). This shift could be attributed to sp-d exchange interactions between the localized $3 \mathrm{~d}$ electrons of $\mathrm{Co}^{+2}$ ions and sp band electrons of the host $\mathrm{ZnO}$ nanocrystals [26, 27]. The obtained results are in good agreement with the results reported by Sakai, Kakeno [28]. The insertion of the cobalt Co ions into the $\mathrm{ZnO}$ structure leads to the appearance of an additional absorption peak [29] at around $408 \mathrm{~nm}$ for all the doped samples.

Using Tauc Plot for calculating the bandgap of Codoped $\mathrm{ZnO}$ nanorod samples where the synthesized nanorods exhibit strong absorption between 374 to $362 \mathrm{~nm}$ according to following equation [30]:

$$
\alpha h v=D\left(h v-E_{g}\right)^{n}
$$

Here, $h v$ is the energy of the incident photon, $E_{g}$, the band gap of the material, $D$ is a constant and $\mathrm{n}$ is the exponent denotes the nature of the transition. The transition data enables us to obtain the best linear fit in the band edge region for $n=1 / 2$ which is referring to direct allowed transition. Plot of $(h v)^{2}$ vs $h v$ is shown in Fig. (3b). By extrapolating the linear portion of the plot to the energy axis, the band gap was calculated and listed in table (1) [31]. The obtained $E_{g}$ values of the investigated samples are varied from 3.016 to $3.152 \mathrm{eV}$ that are smaller than that commonly obtained in the case of bulk $\mathrm{ZnO}$ $(3.37 \mathrm{eV})$. This can be attributed to the high density of the chemical defects and/or vacancies at the intergranular regions that is caused a decrease in the band gap [32]. Moreover, the slightly nonpronounced variation of $E_{g}$ values with Co concentration confirms the substitution of the $\mathrm{Zn}$ sites by Co.

Figure (4) shows XRD patterns of P-CZ0 and P-CZ7 nanocomposite samples as examples. The characteristic diffraction peaks are obtained at $2 \theta=$ $\left(18.99^{\circ} \& 25.2^{\circ}\right)$ and $\left(19.7^{\circ} \& 25.4^{\circ}\right)$ for P-CZ0 and P-CZ7 nanocomposite samples, respectively. Such diffraction peaks are confirming the formation of polyaniline with such diffraction angles due to the parallel and perpendicular periodicity of the PANI chains [33]. The second characteristic diffraction peak at $\left(25.2^{\circ}\right.$ or $\left.25.4^{\circ}\right)$ is more pronounced than the first one at $\left(18.99^{\circ}\right.$ or $\left.19.7^{\circ}\right)$ as shown in Fig. (4). Such result is commonly obtained for highly doped emeraldine PANI [34, 35].

The surface morphologies of the synthesized Codoped $\mathrm{ZnO}$ nanorods as well as PANI nanocomposites were characterized using the transmission high resolution electron microscope (HR-TEM) of the type JEOL-JEM-1230. HR-TEM micrographs of the synthesized Co-doped $\mathrm{ZnO}$ samples are shown in Fig. (5). Such micrographs refer to the formation of Co-doped $\mathrm{ZnO}$ in rod-like shape with reasonable degree of uniformity in the diameter. Moreover, the diameter of the rods is less than $100 \mathrm{~nm}$ that is confirmed the nano-size in one dimensional structure of the investigated particles.
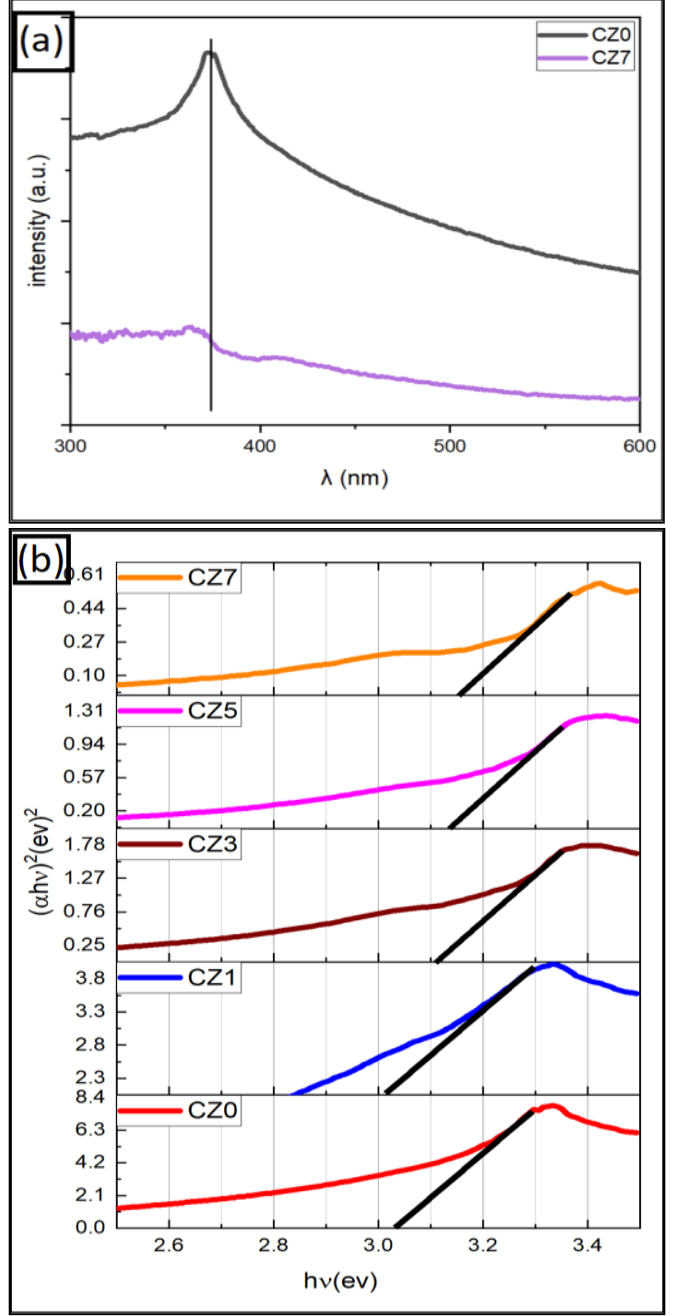

Fig. (3): (a) UV-Visible spectroscopy for CZO and CZ7, (b) Energy gab calculation of Co-doped ZnO nanorod samples 


\section{Belal A. Tahoun, Elsayed M. Farag, Shehab A. Mansour and Maha A. Tony" Development and Characterization of Conjugated Polyaniline/Co doped ZnO Nanocomposites "}

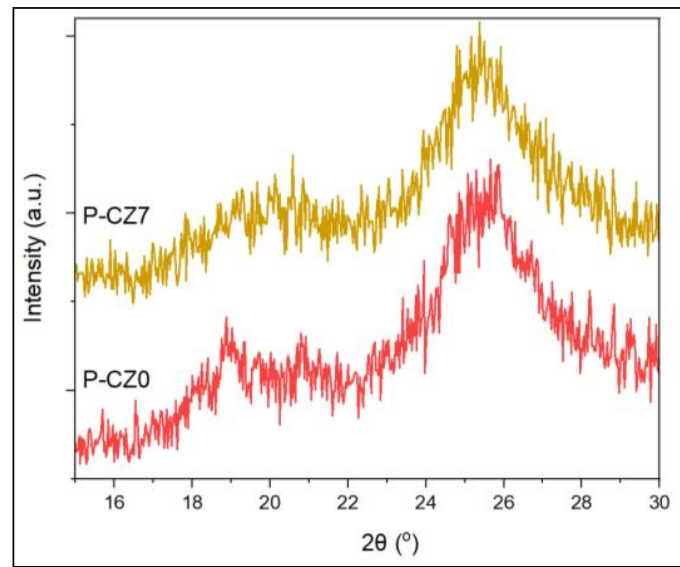

Fig. (4): XRD patterns of P-CZ0 and P-CZ7 nanocomposites

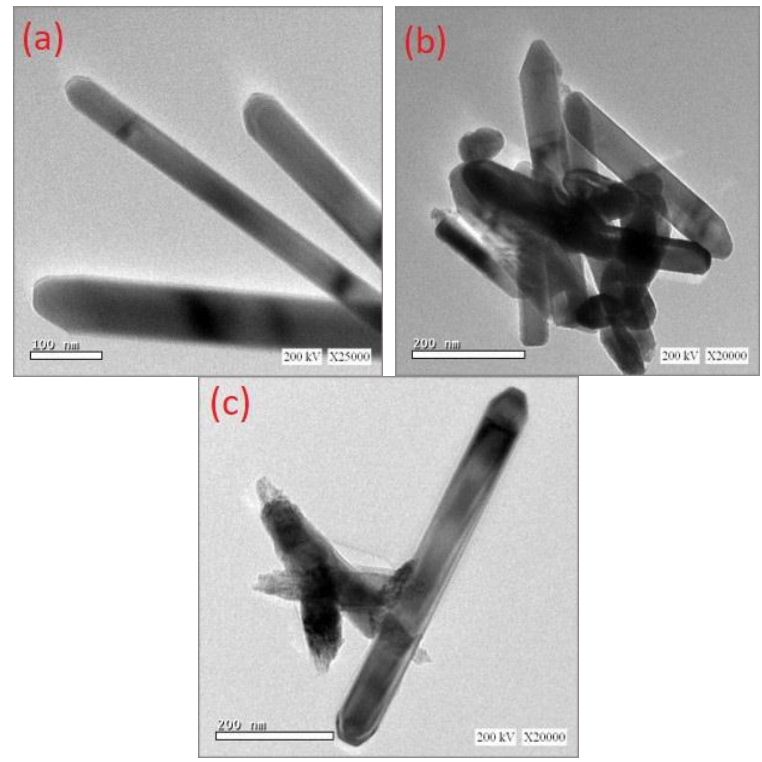

Fig. (5): HR-TEM micrographs of Co-doped $\mathrm{ZnO}$ samples; CZ0 (a), CZ3(b), CZ7(c)

However, HR-TEM micrographs of P-CZ3, PCZ5 nanocomposites are shown in Fig. (6). The PANI matrix displays ribbon-like nanofiber shapes with reasonably dispersion of the Co-doped $\mathrm{ZnO}$ nanorods. Such micrographs confirm the combination between PANI and Co-doped $\mathrm{ZnO}$ nanorods which refers to the importance of ultrasonic radiation in situ of polymerization process of aniline.

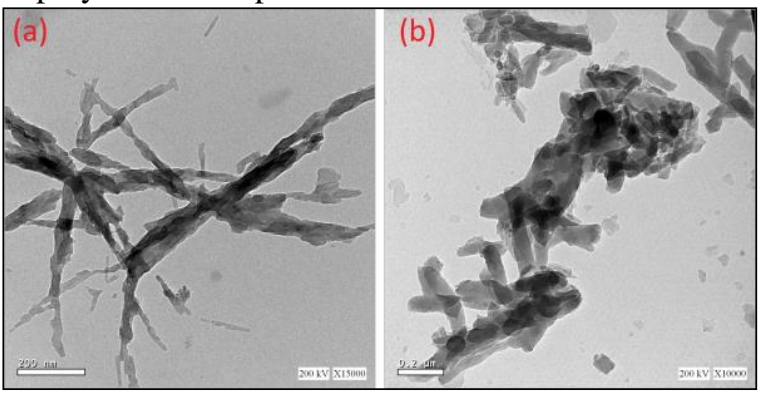

Fig. (6): HR-TEM micrographs of PANI nanocomposites loaded with Co-doped $\mathrm{ZnO}$; (a) PCZ3 \& (b) P-CZ5

\subsection{Catalytic oxidation activity \\ 3.2.1. Effect of circulation flow rate}

In order to investigate the effect of flow rate on the synthesized material activity on aqueous effluent remediation, $\mathrm{MB}$ dye embedded in aqueous stream is treated with $10 \mathrm{ppm}$ MB solution under UV illumination using $20 \mathrm{mg} / \mathrm{L}$ of PANI. Different circulation flow rates ranged from 50 to $147 \mathrm{~mL} / \mathrm{min}$ were applied to investigate the optimal flow rate on the oxidation activity. The results displayed in Fig. (7) assure that increasing the circulation flow rate is increasing the dye removal oxidation efficiency through the concentration removal of dye. The low circulation flow rate causes the catalyst precipitation. Thus, the reaction oxidation rate is reduced. However, the optimal circulation flow rate assures the catalyst suspension in the aqueous effluent and thus maximizing the oxidation rate. This is in accordance with the previous research cited by Tayeb, Tony [6] in treating $\mathrm{MB}$ by $\mathrm{ZnO}$ nanoparticles. Also, previous observations concerning the sour wastewater photo-treatment under photo-Fenton's reagent under artificial UV light reported similar reaction behavior [36]. Hence the optimum flow rate is related to the exposure time to the UV radiation. To add up, the optimal flow rate is also required to attain the conversion of the pollutants to the harmless end products due to the optimal exposure time to the ultraviolet radiation. This investigation is previously reported by Tayeb, Tony [37] in treating basic dye from aqueous solution by hematite nanoparticles.

\subsubsection{Effect of initial dye load}

The load of the initial MB dye concentration in the aqueous stream on its PANI photo-catalytic oxidation (20 mg/L Pure PANI and $147 \mathrm{~mL} / \mathrm{min}$ of circulation flow rate) was illustrated in Fig. (8). The results in Fig. (8) demonstrated that the reaction rate under UV illumination was increased with decreasing the initial dye load and the percentage dye removals are 98.9, $29.6,8.6 \%$ for the initial dye concentrations of 5,10 and $15 \mathrm{ppm}$, respectively. This phenomenon of increasing the MB dye removal rate with the initial dye concentration can be related to the increase in the dye color of the polluted water, which may cause a shadowing effect that prevents the UV illumination from penetration the aqueous solution. Hence, the $\cdot \mathrm{OH}$ radicals decreased and thus, the overall reaction rate was deduced. 


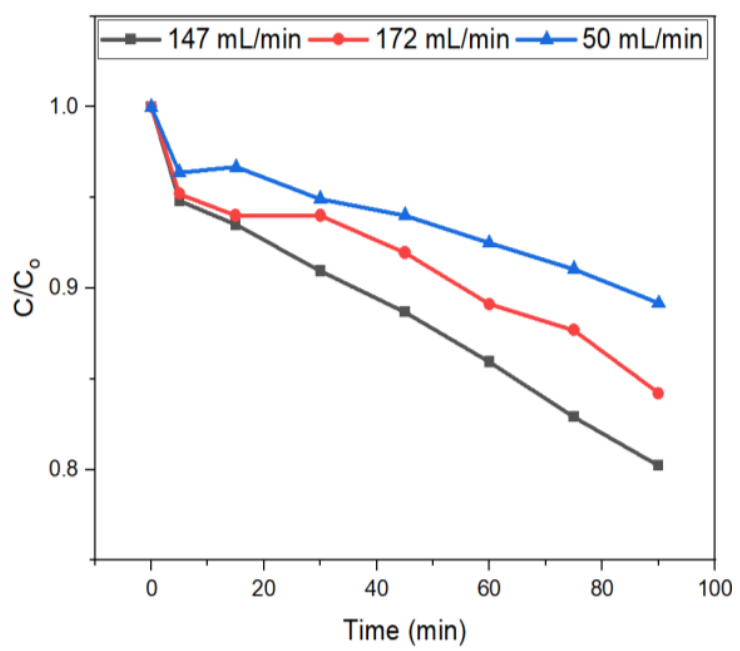

Fig. (7) Effect of Flow rate on the photo oxidation of MB under UV illumination

(PANI catalyst concentration $=20 \mathrm{mg} / \mathrm{L}$, pollutant

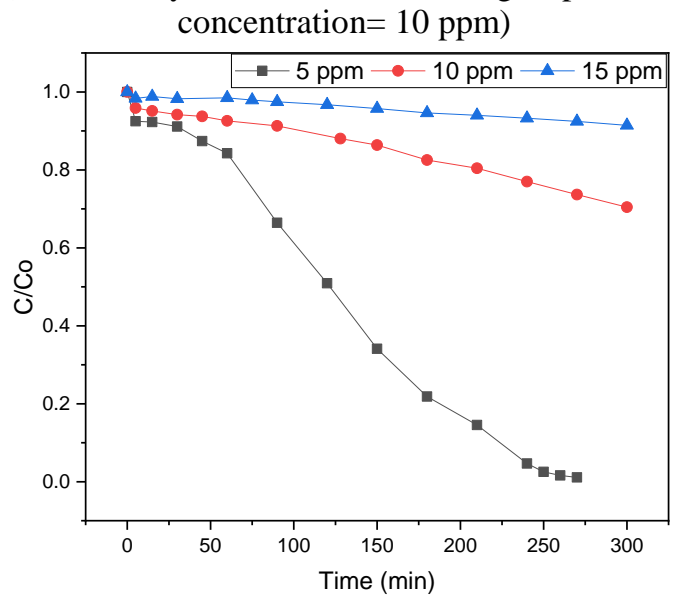

Fig. (8) Effect of initial dye concentration on the photo oxidation of MB under UV illumination (Flow rate $=147 \mathrm{~mL} / \mathrm{min}$, pure PANI catalyst concentration $=20 \mathrm{mg} / \mathrm{L}$ )

Najjar, Chirchi [38] and Tony and Mansour [39] previously recorded the elevation of the photocatalytic oxidation rate with decreasing the initial dye loads in wastewater effluents. Moreover, with the initial MB dye concentration increase, the adsorbed dye molecules on the surface of nanoparticles is also increased, and afterwards the active sites of the catalyst are occupied by the dye molecule. Hence, the oxidation efficiency decreases as the activity of active surface of the nanoparticles decreased. This is due to the reduction in the hydroxyl radicals after the initial time which are considered the main responsible of the reaction [40].

\subsubsection{Effect of $\mathbf{p H}$}

The solution $\mathrm{pH}$ value is considered an effective parameter especially on those reactions that are taking place on the surface of particulate solids such as those on the PANI surface. The value of $\mathrm{pH}$ has its effect on the MB dye molecules oxidation under the UV illumination on the nanoparticles surface. Experiments were run at constant operating parameters (flow rate $=147 \mathrm{~mL} / \mathrm{min}$, MB concentration $=10 \mathrm{ppm}$ and PANI $20 \mathrm{mg} / \mathrm{L}$ ) however; the $\mathrm{pH}$ value is changed as 3, 4, 5, 6 and 7 in order to check the effect of $\mathrm{pH}$ at various values in different ranges.

It is observed from the results shown in Fig. (9) that the rate of oxidation is highest when the applied $\mathrm{pH}$ is 6 . Since the photocatalytic oxidation of MB occurs on the nanoparticles surface is affected by the solution $\mathrm{pH}$. The catalyst behavior can be illustrated by the charge density on nanoparticles surface, which prevails in aqueous medium whereas the $\mathrm{OH}$ radicals production increases [41]. This attitude of the results agrees with the previous work of Zhao, Keogh [42] and Gajbhiye [43]. Nevertheless, elevating the $\mathrm{pH}$ value was unfavorable because undesired radicals were generated rather $\cdot \mathrm{OH}$ radicals which scavenging the reaction yield. This finding of the sensitivity of the reaction $\mathrm{pH}$ to working at a specific limit was previously investigated in the literature [44].

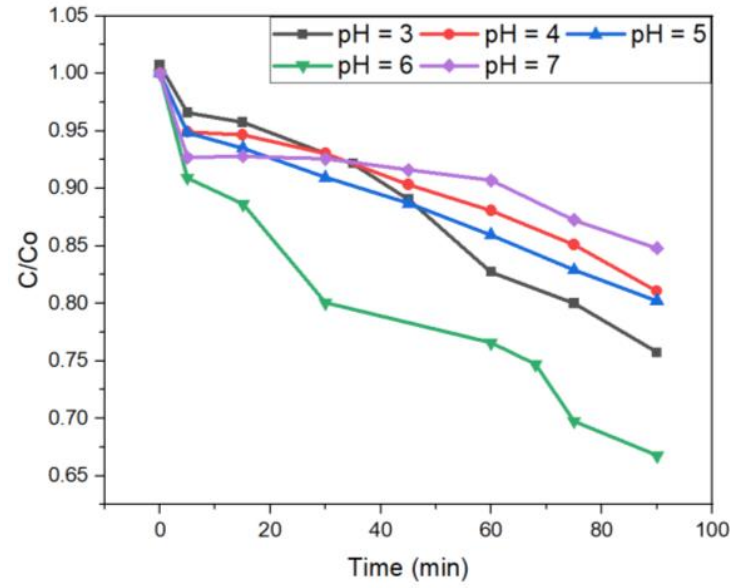

Fig. (9) Effect of $\mathrm{pH}$ on the photo oxidation of $\mathrm{MB}$ under UV illumination

(Flow rate $=147 \mathrm{~mL} / \mathrm{min}$, pollutant concentration $=10$ ppm, Catalyst PANI $=20 \mathrm{mg} / \mathrm{L}$ )

\subsubsection{Effect of catalyst concentration}

A test was run for the decolorization of $\mathrm{MB}$ dye solution at various nanoparticles dosages using solo PANI and the hybrid combination of PANI/Co-doped $\mathrm{ZnO}$ nanocomposite to assure their catalytic activity. Different PANI concentrations $(5-25 \mathrm{mg} / \mathrm{L})$ were undertaken at a constant operating condition to investigate the role of catalyst concentration on the oxidation process during 90 minutes of reaction time. Results in Fig. (10A) showed that increasing pure 


\section{Belal A. Tahoun, Elsayed M. Farag, Shehab A. Mansour and Maha A. Tony" Development and Characterization of Conjugated Polyaniline/Co doped ZnO Nanocomposites}

PANI dose results in an enhancement in the reaction yield and the optimum catalyst concentration was 20 $\mathrm{mg} / \mathrm{L}$. However, increasing the cobalt concentration more than this optimal value reducing the process performance. This finding could be illustrated by the fact that the presence of excess catalyst particles results in the production of more nanoparticles species rather than the more useful $\cdot \mathrm{OH}$ radicals, that are the main responsible of MB removal. Furthermore, the excess catalyst species results in a turbid solution and thus the UV light penetration was deduced. Consequently, the overdosing of nanoparticles in the solution is inhibiting the overall reaction rate [45].

Moreover, five different prepared concentrations of $\mathrm{PANI} /$ pure $\mathrm{ZnO}$ sample (P-CZ0) were applied to check their capabilities for MB removal. The results in Fig. (10B) revealed that the removal efficiency of the particles used in MB dye oxidation is increased with increasing the catalyst concentration and 100 $\mathrm{mg} / \mathrm{L}$ showed a higher removal efficiency. High catalyst dose helps in the presence of more active sites that is

offering enhancement in the interfacial zones between the organic pollutant and catalyst. Moreover, the mount of hydroxyl radicals produced are also higher which are the main responsible of attaching the organics and oxidizing them [3].
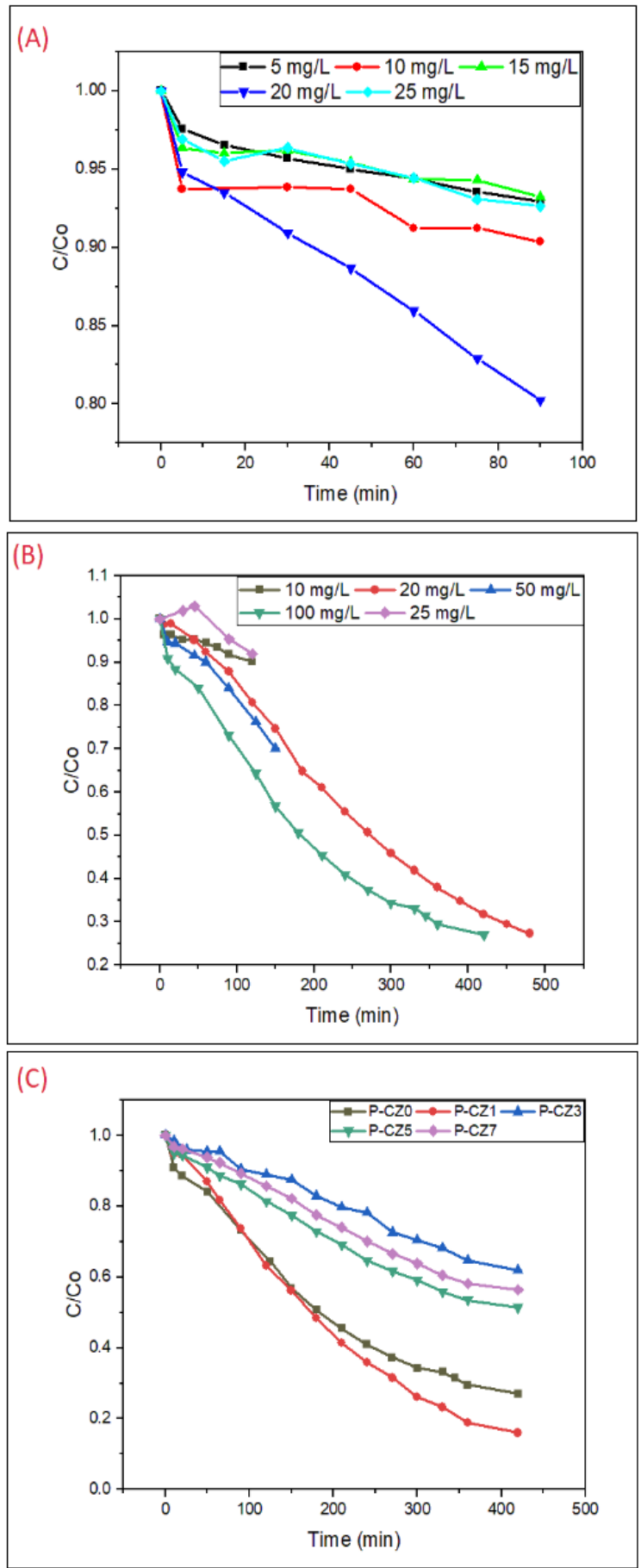

Fig. (10) Effect of different photocatalysts on photocatalytic oxidation for MB under UV light (flow rate $=147 \mathrm{~mL} / \mathrm{min}$, Catalyst dose $=100 \mathrm{mg} / \mathrm{L}$, pollutant concentration $=10 \mathrm{ppm})(\mathrm{A})$ Effect of pure

PANI concentration; (B) Effect of PANI/ZnO nanocomposite sample concentration, and (C) Effect of the investigated PANI/Co-doped $\mathrm{ZnO}$ nanocomposites concentration.

Fig. (10C) illustrates the effect of various PANI/Codoped $\mathrm{ZnO}$ nanocomposites at $100 \mathrm{mg} / \mathrm{L}$ concentration. The results in Fig. (10C) displayed a 


\section{Belal A. Tahoun, Elsayed M. Farag, Shehab A. Mansour and Maha A. Tony" Development and Characterization of Conjugated Polyaniline/Co doped ZnO Nanocomposites}

significant enhancement in the removal of $\mathrm{MB}$ rate was given with the increase of the catalyst dose and the optimal combination is recorded to P-CZ1. This increment in the reaction rate was clearly associated with the $\cdot \mathrm{OH}$ radicals produced since more radicals are produced using different semiconductors. Here it worth to mention that, the combination of PANI, Co and $\mathrm{ZnO}$ results in a higher reaction yield [46].

\section{Conclusions}

PANI nanocomposites loaded by the synthesized Codoped $\mathrm{ZnO}$ nanorods were successfully fabricated via oxidative polymerization route. Photocatalytic oxidation of $\mathrm{MB}$ in aqueous streams was investigated using PANI augmented with Co-doped $\mathrm{ZnO}$ nanorods under UV illumination. Different experimental condition parameters, including, different catalyst type and dosage, flow rate of the aqueous solution, change of initial MB dye concentration, and different $\mathrm{pH}$ values of solutions were evaluated. A suitable operating condition was selected according to the experimental results which revealed that the minimum dye concentration elevating the removal efficiency. $\mathrm{pH} 6.0$ is corresponding to the maximal dye elimination however, $147 \mathrm{~mL} / \mathrm{min}$ is optimal flow rate that equivalent to the maximal organics conversion. PZC1 sample exhibited a reasonable result in treating $\mathrm{MB}$ solution with dye removal reach to $\sim 87 \%$ after $425 \mathrm{~min}$. In conclusion, the obtained results enabled to get a facile fabrication of PANI/ Co-doped $\mathrm{ZnO}$ nanocomposites that are encouraging for usage in the field of wastewater treatment.

\section{References}

[1] M.I. Stefan, Advanced oxidation processes for water treatment: fundamentals and applications. 2017: IWA publishing.

[2] R.H. Thabet, M.K. Fouad, I.A. Ali, S.A. El Sherbiney, and M.A. Tony, Magnetite-based nanoparticles as an efficient hybrid heterogeneous adsorption/oxidation process for reactive textile dye removal from wastewater matrix. International Journal of Environmental Analytical Chemistry, 2021, pp. 1-23.

[3] M.A. Tony, Central composite design optimization of Bismarck Dye oxidation from textile effluent with Fenton's reagent. Applied Water Science, Vol. 10, No. 5, 2020, pp. 1-9.

[4] S.A. Mansour, M.A. Tony, and A.M. Tayeb, Photocatalytic performance and photodegradation kinetics of Fenton-like process based on haematite nanocrystals for basic dye removal. SN Applied Sciences, Vol. 1, No. 3, 2019, pp. 265.
[5] M.A. Tony and S.A. Mansour, Removal of the commercial reactive dye Procion Blue MX7RX from real textile wastewater using the synthesized $\mathrm{Fe} 2 \mathrm{O} 3$ nanoparticles at different particle sizes as a source of Fentons reagent. Nanoscale Advances, Vol. 1, No. 4, 2019, pp. 1362-1371.

[6] A.M. Tayeb, M.A. Tony, and E.K. Ismaeel, Engineered nanostructured $\mathrm{ZnO}$ for water remediation: operational parameters effect, Box-Behnken design optimization and kinetic determinations. Applied Water Science, Vol. 9, No. 3, 2019, pp. 1-11.

[7] M. Kositzi, I. Poulios, S. Malato, J. Caceres, and A. Campos, Solar photocatalytic treatment of synthetic municipal wastewater. Water research, Vol. 38, No. 5, 2004, pp. 11471154.

[8] M.A. Tony and S.A. Mansour, Synthesis of nanosized amorphous and nanocrystalline $\mathrm{TiO} 2$ for photochemical oxidation of methomyl insecticide in aqueous media. Water and Environment Journal, Vol. 34, No. S1, 2020, pp. 239-249.

[9] M. Pirsaheb, S. Moradi, M. Shahlaei, X. Wang, and N. Farhadian, A new composite of nano zero-valent iron encapsulated in carbon dots for oxidative removal of bio-refractory antibiotics from water. Journal of cleaner production, Vol. 209, 2019, pp. 1523-1532.

[10] M.A. Tony and L.-S. Lin, Iron Coated-Sand from Acid Mine Drainage Waste for Being a Catalytic Oxidant Towards Municipal Wastewater Remediation. International Journal of Environmental Research, Vol. 15, No. 1, 2021, pp. 191-201.

[11] M. Umar, H.A.J.O.p.-m. Aziz, risk, and treatment, Photocatalytic degradation of organic pollutants in water. Vol. 8, 2013, pp. 196-197.

[12] A. Hatamie, A. Khan, M. Golabi, A.P. Turner, V. Beni, W.C. Mak, A. Sadollahkhani, H. Alnoor, B. Zargar, and S.J.L. Bano, Zinc oxide nanostructure-modified textile and its application to biosensing, photocatalysis, and as antibacterial material. Vol. 31, No. 39, 2015, pp. 10913-10921.

[13] P. Nagajyothi, T.M. An, T. Sreekanth, J.-i. Lee, D.J. Lee, and K.J.M.L. Lee, Green route biosynthesis: Characterization and catalytic activity of $\mathrm{ZnO}$ nanoparticles. Vol. 108, 2013, pp. 160-163. 


\section{Belal A. Tahoun, Elsayed M. Farag, Shehab A. Mansour and Maha A. Tony" Development and Characterization of Conjugated Polyaniline/Co doped ZnO Nanocomposites}

[14] S.P. Sharma, M. Suryanarayana, A.K. Nigam, A. Chauhan, and L.J.C.C. Tomar, [PANI/ZnO] composite: Catalyst for solvent-free selective oxidation of sulfides. Vol. 10, No. 6, 2009, pp. 905-912.

[15] S.P. Ansari and F.J.I.M.S. Mohammad, Studies on nanocomposites of polyaniline and zinc oxide nanoparticles with supporting matrix of polycarbonate. Vol. 2012, 2012.

[16] M. Mitra, A. Ghosh, A. Mondal, K. Kargupta, S. Ganguly, and D. Banerjee, Facile synthesis of aluminium doped zinc oxide-polyaniline hybrids for photoluminescence and enhanced visible-light assisted photo-degradation of organic contaminants. Applied Surface Science, Vol. 402, 2017, pp. 418-428.

[17] V. Eskizeybek, F. Sarı, H. Gülce, A. Gülce, and A. Avc1, Preparation of the new polyaniline/ $\mathrm{ZnO}$ nanocomposite and its photocatalytic activity for degradation of methylene blue and malachite green dyes under UV and natural sun lights irradiations. Applied Catalysis B: Environmental, Vol. 119, 2012, pp. 197-206.

[18] M. Samadi, M. Zirak, A. Naseri, E. Khorashadizade, and A.Z. Moshfegh, Recent progress on doped $\mathrm{ZnO}$ nanostructures for visible-light photocatalysis. Thin Solid Films, Vol. 605, 2016, pp. 2-19.

[19] V. Gilja, I. Vrban, V. Mandić, M. Zic, and Z. Hrnjak-Murgić, Preparation of a PANI/ZnO Composite for Efficient Photocatalytic Degradation of Acid Blue. Polymers, Vol. 10, 2018, pp. 940.

[20] A.H. Farha, S.A. Mansour, and M.F. Kotkata, Structural, optical, and magnetic study of dilute magnetic semiconducting Co-doped $\mathrm{ZnO}$ nanocrystals synthesized using polymerpyrolysis route. Journal of Materials Science, Vol. 51, No. 21, 2016, pp. 9855-9864.

[21] P. Scherrer, Bestimmung der Große und der inneren Struktur von Kolloidteilchen mittels Rontgenstrahlen. Nachrichten von der Gesellschaft der Wissenschaften zu Gottingen, Mathematisch-Physikalische Klasse, Vol. 1918, 1918, pp. 98-100.

[22] A.L. Patterson, The Scherrer Formula for XRay Particle Size Determination. Physical Review, Vol. 56, No. 10, 1939, pp. 978-982.

[23] J.I. Langford and A.J.C. Wilson, Scherrer after sixty years: A survey and some new results in the determination of crystallite size. Journal of Applied Crystallography, Vol. 11, No. 2, 1978, pp. 102-113.

[24] B. Cheng and E.T. Samulski, Hydrothermal synthesis of one-dimensional $\mathrm{ZnO}$ nanostructures with different aspect ratios. Chemical Communications, No. 8, 2004, pp. 986-987.

[25] A. Kaushik, B. Dalela, R. Rathore, V.S. Vats, B.L. Choudhary, P.A. Alvi, S. Kumar, and S. Dalela, Influence of Co doping on the structural, optical and magnetic properties of $\mathrm{ZnO}$ nanocrystals. Journal of Alloys and Compounds, Vol. 578, 2013, pp. 328-335.

[26] M. Nirmala and A. Anukaliani, Characterization of undoped and $\mathrm{Co}$ doped $\mathrm{ZnO}$ nanoparticles synthesized by DC thermal plasma method. Physica B: Condensed Matter, Vol. 406, No. 4, 2011, pp. 911-915.

[27] T.M. Hammad, J.K. Salem, and R.G. Harrison, Structure, optical properties and synthesis of Co-doped $\mathrm{ZnO}$ superstructures. Applied Nanoscience, Vol. 3, No. 2, 2012, pp. 133139.

[28] K. Sakai, T. Kakeno, T. Ikari, S. Shirakata, T. Sakemi, K. Awai, and T. Yamamoto, Defect centers and optical absorption edge of degenerated semiconductor $\mathrm{ZnO}$ thin films grown by a reactive plasma deposition by means of piezoelectric photothermal spectroscopy. Journal of Applied Physics, Vol. 99, No. 4, 2006, pp. 043508-043508.

[29] A. Mesaros, C.D. Ghitulica, M. Popa, R. Mereu, A. Popa, T. Petrisor, M. Gabor, A.I. Cadis, and B.S. Vasile, Synthesis, structural and morphological characteristics, magnetic and optical properties of $\mathrm{Co}$ doped $\mathrm{ZnO}$ nanoparticles. Ceramics International, Vol. 40, No. 2, 2014, pp. 2835-2846.

[30] P.K. Samanta, A. Saha, and T. Kamilya, Chemical synthesis and optical properties of $\mathrm{ZnO}$ nanoparticles. Журнал нано-та електронної фізики, №. 6,№ 4, 2014, pp. 04015-1.

[31] A. Murali and H.Y. Sohn, Photocatalytic properties of plasma-synthesized aluminumdoped zinc oxide nanopowder. Journal of nanoscience and nanotechnology, Vol. 19, No. 8, 2019, pp. 4377-4386.

[32] A.H. Farha, M.M. Ibrahim, and S.A. Mansour, Ga-doped $\mathrm{ZnO}$ nanostructured powder for cool- 


\section{Belal A. Tahoun, Elsayed M. Farag, Shehab A. Mansour and Maha A. Tony" Development and Characterization of Conjugated Polyaniline/Co doped ZnO Nanocomposites}

nanopigment in environment applications. Materials, Vol. 13, No. 22, 2020, pp. 5152.

[33] A. Mostafaei and A. Zolriasatein, Synthesis and characterization of conducting polyaniline nanocomposites containing $\mathrm{ZnO}$ nanorods. Progress in Natural Science: Materials International, Vol. 22, No. 4, 2012, pp. 273280.

[34] J.P. Pouget, M.E. Jozefowicz, A.J.e.a. Epstein, X. Tang, and A.G. MacDiarmid, X-ray structure of polyaniline. Macromolecules, Vol. 24, No. 3, 1991, pp. 779-789.

[35] H. Xia and Q. Wang, Ultrasonic irradiation: a novel approach to prepare conductive polyaniline/nanocrystalline titanium oxide composites. Chemistry of Materials, Vol. 14, No. 5, 2002, pp. 2158-2165.

[36] D. Sareen, R. Garg, and N. Grover, A study on removal of methylene blue dye from waste water by adsorption technique using fly ash briquette. International Journal, Vol. 5, No. 4, 2014.

[37] A.M. Tayeb, M.A. Tony, and S.A. Mansour, Application of Box-Behnken factorial design for parameters optimization of basic dye removal using nano-hematite photo-Fenton tool. Applied Water Science, Vol. 8, No. 5, 2018, pp. 1-9.

[38] W. Najjar, L. Chirchi, E. Santos, and A. Ghorhel, Kinetic study of 2-nitrophenol photodegradation on Al-pillared montmorillonite doped with copper. Journal of Environmental Monitoring, Vol. 3, No. 6, 2001, pp. 697-701.

[39] M.A. Tony and S.A. Mansour, Removal of the commercial reactive dye Procion Blue MX$7 \mathrm{RX}$ from real textile wastewater using the synthesized Fe 2 O 3 nanoparticles at different particle sizes as a source of Fenton's reagent. Nanoscale Advances, Vol. 1, No. 4, 2019, pp. 1362-1371.

[40] E.M. Saggioro, A.S. Oliveira, T. Pavesi, C.G. Maia, L.F.V. Ferreira, and J.C. Moreira, Use of titanium dioxide photocatalysis on the remediation of model textile wastewaters containing azo dyes. Molecules, Vol. 16, No. 12, 2011, pp. 10370-10386.

[41] M. El Haddad, A. Regti, M.R. Laamari, R. Mamouni, and N. Saffaj, Use of Fenton reagent as advanced oxidative process for removing textile dyes from aqueous solutions. J Mater Environ Sci, Vol. 5, No. 3, 2014, pp. 667674.

[42] Y.Q. Zhao, C. Keogh, and M.A. Tony, On the necessity of sludge conditioning with nonorganic polymer: AOP approach. Journal of Residuals Science \& Technology, Vol. 6, No. 3, 2009, pp. 151-155.

[43] S.B. Gajbhiye, Photocatalytic degradation study of methylene blue solutions and its application to dye industry effluent. Int. J. Mod. Eng. Res, Vol. 2, No. 3, 2012, pp. 1204-1208.

[44] M.A. Tony and L.-S. Lin, Iron recovery from acid mine drainage sludge as Fenton source for municipal wastewater treatment. International Journal of Environmental Analytical Chemistry, 2020, pp. 1-16.

[45] J. Rodríguez-Chueca, M.I. Polo-López, R. Mosteo, M.P. Ormad, and P. Fernandez-Ibanez, Disinfection of real and simulated urban wastewater effluents using a mild solar photoFenton. Applied Catalysis B: Environmental, Vol. 150, 2014, pp. 619-629.

[46] S.A. Kamaruddin, K.-Y. Chan, H.-K. Yow, M.Z. Sahdan, H. Saim, and D. Knipp, Zinc oxide films prepared by sol-gel spin coating technique. Applied Physics A, Vol. 104, No. 1, 2011, pp. 263-268. 